\title{
A WEAK GAGA STATEMENT FOR ARBITRARY MORPHISMS
}

\author{
AMNON NEEMAN
}

\begin{abstract}
Let $f: X \rightarrow Y$ be an arbitrary morphism of schemes of finite type over $\mathbf{C}$, and let $f^{\text {an }}$ be the associated map of analytic spaces. Let $\mathscr{S}$ be a coherent sheaf on $X$. Then $\left(f_{*} \mathscr{S}\right)^{\text {an }} \rightarrow f_{*}^{\text {an }}\left(\mathscr{S}^{\text {an }}\right)$ is injective.
\end{abstract}

0. Introduction. Serre's paper GAGA, as generalized by Gorthendieck, proves the following result. Let $X$ and $Y$ be schemes of finite type over C. Let $f: X \rightarrow Y$ be a proper morphism. Let $\mathscr{S}$ be a coherent sheaf on $X$. Then if an stands for the analytification functor, the natural map $\phi:\left(R^{i} f_{*} \mathscr{S}\right)^{\text {an }} \rightarrow R^{i} f_{*}^{\text {an }}\left(\mathscr{S}^{\text {an }}\right)$ is an isomorphism. It is well known that this breaks down if $f$ is not proper.

In this note, I prove that one can rescue an infinitesimal amount in the general case. If $f: X \rightarrow Y$ is arbitrary, I prove that $\left(f_{*} \mathscr{S}\right)^{\text {an }} \rightarrow f_{*}^{\text {an }} \mathscr{S}^{\text {an }}$ is injective.

One application of this result is to give an alternative approach to my proof of injectivity in Neeman [N, Lemmas 4.7 and 4.8 and Proposition 4.9]. One way to view the original proof is as a trick that gets around the theory developed here.

1. Preliminaries. In this section I want to review some of the definitions in Serre's paper (Serre [GAGA]). Let $X$ be a scheme of finite type over C. Serre defined what is meant by the analytification $X^{\text {an }}$ of $X$ (Serre denotes that space $X^{h}$ ). He also defined what is meant by the analytification of an algebraic sheaf on $X$. Allow me to recall the definitions so as to remind the reader of the basic setup.

There is an underlying continuous map of ringed topological spaces $X^{\text {an }} \rightarrow X$. Let $\mathscr{S}$ be a sheaf of $\mathcal{O}_{X}$-modules on $X$. The analytification $\mathscr{S}^{\text {an }}$ of $\mathscr{S}$ is defined to be the sheaf $\phi^{-1} \mathscr{S} \otimes_{\phi^{-1} \mathcal{O}_{X}} \mathcal{O}_{X^{\text {an }}}$.

REMARK 1.1. $\mathscr{S}^{\text {an }}$ is a sheaf of $\mathcal{O}_{X^{\text {an }}}$-modules on $X^{\text {an }}$. The map $\mathscr{S} \mapsto \mathscr{S}^{\text {an }}$ extends to a functor from the category of $\mathcal{O}_{X}$ modules over $X$ to the category of $\mathcal{O}_{X^{\text {an- }}}$ modules on $X^{\text {an }}$. We also denote this functor by $\phi^{*}$.

REMARK 1.2. The functor $\phi^{*}$ is left adjoint to the functor $\phi_{*}$ (viewed as a functor from the category of $\mathcal{O}_{X}$ an-modules to the category of $\mathcal{O}_{X}$-modules).

REMARK 1.3. The stalk of $\mathscr{S}^{\text {an }}$ at a point $x \in X^{\text {an }}$ is given by the formula $\mathscr{S}_{x}^{\text {an }}=S_{x} \otimes_{\mathcal{O}_{X, x}} \mathcal{O}_{X^{\text {an }}, x}$.

REMARK 1.4. Analytification is an exact functor. This can be checked on stalks, so it follows from Remark 1.3 (cf. Serre [GAGA, §3, Proposition 10]).

Received by the editors April 22, 1986.

1980 Mathematics Subject Classification (1985 Revision). Primary 14F99; Secondary 32C15. 
Let $f: X \rightarrow Y$ be a morphism of schemes. One defines $f^{\text {an }}: X^{\text {an }} \rightarrow Y^{\text {an }}$, and easily verifies that the following diagram of continuous maps of ringed topological spaces commutes:

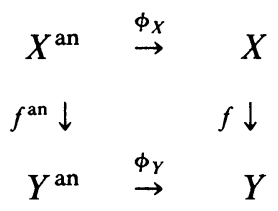

On $X$, we have a natural map $f^{*} f_{*} \mathscr{S} \rightarrow \mathscr{S}$. We deduce a map $\phi_{X}^{*} f^{*} f_{*} \mathscr{S} \rightarrow \phi_{X}^{*} \mathscr{S}$. By the commutativity of the diagram, there is a natural isomorphism $\phi_{X}^{*} f^{*} \cong\left(f^{\text {an }}\right)^{*} \phi_{Y}^{*}$. So we deduce a map $\left(f^{\text {an }}\right)^{*} \phi_{Y}^{*} f_{*} \mathscr{S} \rightarrow \phi_{X}^{*} \mathscr{S}$. Finally, we get a map by adjunction $\phi_{Y}^{*} f_{*} \mathscr{S} \rightarrow f_{*}^{\text {an }} \phi_{X}^{*} \mathscr{S}$. In other words, there is a natural map $\phi:$ $\left(f_{*} \mathscr{S}\right)^{\text {an }} \rightarrow f_{*}^{\text {an }}\left(\mathscr{S}^{\text {an }}\right)$.

REMARK 1.5. A fact that we will not need in this paper is that this natural comparison map can be extended to compare the higher derived functors of $f_{*}$. Precisely, observe that because analytification is exact, the functors $\mathscr{S} \mapsto\left(R^{i} f_{*} \mathscr{S}\right)^{\text {an }}$ are the higher derived functors of $\mathscr{S} \mapsto\left(f_{*} \mathscr{S}\right)^{\text {an }}$. Because $\mathscr{S} \mapsto R^{i} f_{*}^{\text {an }}\left(\mathscr{S}^{\text {an }}\right)$ is also a $\delta$-functor (again, we use the exactness of analytification), it follows that there is a unique natural comparison map of $\delta$-functors $\left(R^{i} f_{*} \mathscr{S}\right)^{\text {an }} \rightarrow R^{i} f_{*}^{\text {an }}\left(\mathscr{S}^{\text {an }}\right)$.

REMARK 1.6. If $\mathscr{S}$ is a coherent sheaf on $X, \mathscr{S}^{\text {an }}$ is a coherent sheaf on $X^{\text {an }}$.

2. The weak GAGA theorem. We begin by proving

LEMMA 2.1. Let $X$ be an affine scheme of finite type over $\mathbf{C}$. Let $\mathscr{S}$ be a coherent algebraic sheaf on $X$. Let $U$ be a Stein open subset of $X^{\text {an }}$. Then the natural map $\Gamma(X, \mathscr{S}) \otimes_{\Gamma\left(X, \mathcal{O}_{X}\right)} \Gamma\left(U, \mathcal{O}_{X^{\text {an }}}\right) \rightarrow \Gamma\left(U, \mathscr{S}^{\text {an }}\right)$ is an isomorphism.

Proof. The lemma is clear if $\mathscr{S} \cong \mathcal{O}_{X}$. But because $\mathscr{S}$ is coherent on the affine scheme $X$, it admits a resolution $\mathcal{O}_{X}^{m} \rightarrow \mathcal{O}_{X}^{n} \rightarrow \mathscr{S} \rightarrow 0$. Analytification is exact, and because $U$ is Stein, the functor sending coherent sheaves on $U$ to their global sections is also exact. It follows that the rows in the commutative diagram below are exact:

$$
\begin{aligned}
& \Gamma\left(X, \mathcal{O}_{X}^{m}\right) \otimes_{\Gamma\left(X, \mathcal{O}_{X}\right)} \Gamma\left(U, \mathcal{O}_{X^{\text {an }}}\right) \rightarrow \Gamma\left(U, \mathcal{O}_{X}^{n}\right) \otimes_{\Gamma\left(X, \mathcal{O}_{X}\right)} \Gamma\left(U, \mathcal{O}_{X^{\mathrm{an}}}\right) \rightarrow \Gamma(X, \mathscr{S}) \otimes_{\Gamma\left(X, \mathcal{O}_{X}\right)} \Gamma\left(U, \mathcal{O}_{X^{\mathrm{an}}}\right) \rightarrow 0 \\
& \begin{array}{ccc}
\mid \downarrow & \mid \downarrow & \downarrow \\
\Gamma\left(U,\left(\mathcal{O}_{X}^{\text {an }}\right)^{m}\right) & \rightarrow \Gamma\left(U,\left(\mathcal{O}_{X}^{\text {an }}\right)^{n}\right) & \rightarrow \Gamma\left(U, \mathscr{S}^{\text {an }}\right)
\end{array}
\end{aligned}
$$

From this the lemma is immediate. Q.E.D.

Next we prove

LEMMA 2.2. Let $X$ and $Y$ be affine schemes of finite type over $C$. Then, for any $y \in Y$, the ring

$$
\lim _{\substack{U \text { open in } \\ y \in U}} \Gamma\left(X^{\text {an }} \text { an } \times U, \mathcal{O}_{X^{\text {an }} \times Y^{\text {an }}}\right)
$$

is faithfully flat over $\Gamma\left(X, \mathcal{O}_{X}\right) \otimes_{\mathrm{C}} \mathcal{O}_{Y^{\mathrm{an}}, y}$. 
Proof. First we prove flatness. Let $I$ be an ideal of $\Gamma\left(X, \mathcal{O}_{X}\right) \otimes_{\mathbf{C}} \mathcal{O}_{Y^{\text {an }}, y}$. $\Gamma\left(X, \mathcal{O}_{X}\right) \otimes_{C} \mathcal{O}_{Y^{\text {an }}, y}$ is noetherian (it is finitely generated algebra over $\mathcal{O}_{Y^{\text {an }}, y}$ ), so $I$ is a finitely presented ideal. Now we want to stretch this to a little neighborhood of $y$ in $Y^{\text {an }}$.

Let $\bar{X}$ be a projective scheme over $\mathbf{C}$ such that $X \subset \bar{X}$ is an open immersion and $\bar{X}-X$ is the vanishing of one section $w \in \Gamma(\bar{X}, \mathcal{O}(1))$ (i.e. compactify $X$ to $\bar{X}$ by adding a divisor). Then the ideal $I$ in $\Gamma\left(X, \mathcal{O}_{X}\right) \otimes_{C} \mathcal{O}_{Y^{\text {an }}, y}$ extends to an ideal sheaf $\mathscr{J}$ on $\bar{X} \times{ }_{\text {Spec C }} \operatorname{Spec} \mathcal{O}_{Y^{\text {an }}, y}$. We can resolve the ideal sheaf $\mathscr{J}:$ precisely, we get a resolution $\mathcal{O}\left(-n_{1}\right)^{r_{1}} \rightarrow \mathcal{O}\left(-n_{2}\right)^{r_{2}} \rightarrow \mathscr{J}$ of $\mathscr{J}$. Because $\bar{X}^{\text {an }}$ is compact, we can stretch the resolution a little in the $Y$-direction; there exists $U \subset Y$ open, an ideal sheaf $\mathscr{J}^{\text {an }}$ on $\bar{X}^{\text {an }} \times U$ and a resolution $\mathcal{O}\left(-n_{1}\right)^{r_{1}} \rightarrow \mathcal{O}\left(-n_{2}\right)^{r_{2}} \rightarrow \mathscr{J}^{\text {an }}$. If we extend $I$ to $\Gamma\left(X, \mathcal{O}_{X}\right) \otimes_{C} \Gamma\left(U, \mathcal{O}_{Y^{\text {an }}}\right)$ by defining it to be the quotient of the corresponding map $\Gamma\left(X, \mathcal{O}_{X}\right) \otimes_{C} \Gamma\left(U, \mathcal{O}_{Y^{a n}}\right)^{r_{1}} \rightarrow \Gamma\left(X, \mathcal{O}_{X}\right) \otimes_{C} \Gamma\left(U, \mathcal{O}_{Y^{a n}}\right)^{r_{2}}$, then by an argument exactly parallel to that of Lemma 2.1 , we deduce that, provided $U$ is a Stein space, the natural map

$$
I \otimes_{\Gamma\left(X, \mathcal{O}_{X}\right) \otimes_{\mathrm{C}} \Gamma\left(U, \mathcal{O}_{Y^{\text {an }}}\right)} \Gamma\left(X^{\text {an }} \times U, \mathcal{O}_{X^{\mathrm{an}} \times Y^{\text {an }}}\right) \rightarrow \Gamma\left(X^{\text {an }} \times U, \mathscr{J}^{\text {an }}\right)
$$

is an isomorphism. But the map $\Gamma\left(X^{\text {an }} \times U, \mathscr{J}^{\text {an }}\right) \rightarrow \Gamma\left(X^{\text {an }} \times U, \mathcal{O}_{X^{\text {an }} \times Y^{\text {an }}}\right)$ is injective. Shrinking $U$ and taking direct limits, the flatness we asserted follows.

Next, we prove faithful flatness. Suppose $f_{1}, \ldots, f_{r} \in \Gamma\left(X, \mathcal{O}_{X}\right) \otimes_{C} \mathcal{O}_{Y^{\text {an }}, y}$ generate the unit ideal in $\lim _{\rightarrow} \Gamma\left(X^{\text {an }} \times U, \mathcal{O}_{X^{\text {an }} \times Y^{\text {an }}}\right)$. We have to show that they already generate the unit ideal in $\Gamma\left(X, \mathcal{O}_{X}\right) \otimes_{\mathrm{C}} \mathcal{O}_{Y^{\text {an }}, y}$.

For small enough $U, f_{1}, \ldots, f_{r}$ already lift to elements of $\Gamma\left(X^{\text {an }} \times U, \mathcal{O}_{X^{\text {an }}, Y^{\text {an }}}\right)$. Shrinking $U$, we may assume that they generate the unit ideal in $\Gamma\left(X^{\text {an }} \times\right.$

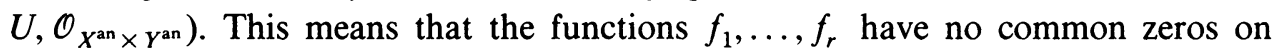
$X^{\text {an }} \times U$.

We compactify $X$ to $\bar{X}$ as before. For some $n$ sufficiently large, $f_{1}, \ldots, f_{r}$ extend to sections of $\Gamma\left(\bar{X}^{\text {an }} \times U, \mathcal{O}(n)^{\text {an }}\right)$, at least if we shrink $U$ suitably (unless we shrink $U$, the degree of the $f_{i}$ 's may become unbounded).

The fact that $f_{1}, \ldots, f_{r}$ have no common zero on $X^{\text {an }} \times U$ can be restated as saying that the cokernel of the corresponding map $\left[\mathcal{O}(-n)^{\mathrm{an}}\right]^{r} \rightarrow \mathcal{O}^{\text {an }}$ is supported on $(\bar{X}-X)^{\text {an }} \times U$. Shrinking $U$ some more, the cokernel will be annihilated by $w^{m}$ for some $m>0$.

Twisting by a large $N$ and shrinking $U$ some more to a small Stein space, we can ensure that the map $\Gamma\left(\bar{X}^{\text {an }} \times U,\left[\mathcal{O}(-n+N)^{\text {an }}\right]^{r}\right) \rightarrow \Gamma\left(\bar{X}^{\text {an }} \times U, \mathcal{O}(N)^{\text {an }}\right)$ will surject to the kernel of the map to the quotient sheaf. If $N$ is chosen $\geqslant m, w^{N}$ lies in the image.

Reading this on $X \subset \bar{X}$, we see that we have expressed 1 as a $\Gamma\left(X, \mathcal{O}_{X}\right) \otimes_{\mathbf{C}}$ $\Gamma\left(U, \mathcal{O}_{Y^{\text {an }}}\right)$ linear confirmation of the $f_{i}$ 's.

(I have slurred over several points here. For instance, we are using the usual GAGA applied to the projective space $\bar{X}$, to deduce that $\Gamma(\bar{X}, \mathcal{O}(p)) \rightarrow$ $\Gamma\left(\bar{X}^{\text {an }}, \mathcal{O}(p)^{\text {an }}\right)$ is an isomorphism. We are actually using a slight generalization; we need to do this on $\bar{X} \times U$. I leave it to the reader to complete the proof in these details.) Q.E.D. 
Lemma 2.3. Let $X$ and $Y$ be affine schemes of finite type over C. Let $\pi: X \times Y \rightarrow Y$ be the projection. If $\mathscr{S}$ is a coherent sheaf on $X \times Y, \phi:\left(\pi_{*} \mathscr{S}\right)^{\text {an }} \rightarrow \pi_{*}^{\text {an }}\left(\mathscr{S}^{\text {an }}\right)$ is injective.

Proof. This can be studied on stalks at $y \in Y$. The stalk of the first sheaf is

$$
\begin{aligned}
& \lim _{y \in U \subset Y^{\text {an }}} \Gamma(X \times Y, \mathscr{S}) \otimes_{\Gamma\left(Y, \mathcal{O}_{Y}\right)} \Gamma\left(U, \mathcal{O}_{Y^{\text {an }}}\right) \\
& \cong \Gamma(X \times Y, \mathscr{S}) \otimes_{\Gamma\left(X, \mathcal{O}_{X}\right) \otimes_{\mathrm{C}} \Gamma\left(Y, \mathcal{O}_{Y}\right)} \Gamma\left(X, \mathcal{O}_{X}\right) \otimes_{\mathrm{C}} \mathcal{O}_{Y^{\text {an }}, y} .
\end{aligned}
$$

The stalk of the second sheaf is

$$
\begin{aligned}
\lim _{y \in U \subset Y^{\text {an }}} \Gamma\left(X^{\text {an }} \times U, \mathscr{S}\right) \\
\quad \cong \lim _{y \in U \subset Y}^{\rightarrow} \Gamma(X \times Y, \mathscr{S}) \otimes_{\Gamma\left(X, \mathcal{O}_{X}\right) \otimes_{\mathrm{c}} \Gamma\left(Y, \mathcal{O}_{Y}\right)} \Gamma\left(X{ }^{\mathrm{an}} \times U, \mathcal{O}_{X^{\mathrm{an}} \times Y^{\mathrm{an}}}\right)
\end{aligned}
$$

(the isomorphism is a consequence of Lemma 2.1). The natural map is injective by the faithful flatness assertion of Lemma 2.2. Q.E.D.

LEMMA 2.4. Let $f: X \rightarrow Y$ be a morphism of affine schemes of finite type over $\mathbf{C}$. Let $\mathscr{S}$ be a coherent sheaf on $X$. Then $\phi:\left(f_{*} \mathscr{S}\right)^{\text {an }} \rightarrow f_{*}^{\text {an }}\left(\Sigma^{\text {an }}\right)$ is injective.

Proof. Express $f$ as the composite of a closed immersion and a projection (i.e. $X \stackrel{i}{\rightarrow} X \times Y \stackrel{\pi}{\rightarrow} Y$, the first map being the graph). The $\phi$ is the composite $\left(\pi_{*} i_{*} \mathscr{S}\right)^{\text {an }}$ $\rightarrow \pi_{*}^{\text {an }}\left(i_{*} \Sigma\right)^{\text {an }} \rightarrow \pi_{*}^{\text {an }} i_{*}^{\text {an }} \mathscr{S}^{\text {an }}$. The first map is injective by Lemma 2.3 , the second is an isomorphism (analytification commutes with closed immersions). Q.E.D.

Finally, we prove

THEOREM 2.5. Let $f: X \rightarrow Y$ be an arbitrary morphism of schemes of finite type over C. Let $\mathscr{S}$ be a coherent sheaf on $X$. Then $\phi:\left(f_{*} \mathscr{S}\right)^{\text {an }} \rightarrow f_{*}^{\text {an }}\left(\mathscr{S}^{\text {an }}\right)$ is injective.

Proof. The problem is local in $Y$, so we may assume $Y$ affine. Cover $X$ by affines $X_{i}, i \in I$, where $I$ is a finite set. Then if $\left.f\right|_{X_{i}}=f_{i},\left[\left(f_{i}\right)_{*} \mathscr{S}\right]^{\text {an }} \rightarrow\left(f_{i}^{\text {an }}\right)_{*} \mathscr{S}^{\text {an }}$ is injective by Lemma 2.4. Hence $\prod_{i \in I}\left[\left(f_{i}\right)_{*} \mathscr{S}\right]^{\text {an }} \rightarrow \prod_{i \in I}\left(f_{i}^{\text {an }}\right)_{*}\left(\mathscr{S}^{\text {an }}\right)$ is injective. It follows that $\phi:\left(f_{*} \mathscr{S}\right)^{\text {an }} \rightarrow f_{*}^{\text {an }}\left(\mathscr{S}^{\text {an }}\right)$ is an injection, because $\left(f_{*} \mathscr{S}\right)^{\text {an }}$ is a subsheaf of $\prod_{i \in I}\left[\left(f_{i}\right)_{*} \mathscr{S}\right]^{\text {an }}$, while $f_{*}^{\text {an }} \mathscr{S}^{\text {an }}$ is a subsheaf of $\prod_{i \in I}\left(f_{i}^{\text {an }}\right)_{*}\left(\mathscr{S}^{\text {an }}\right)$. Q.E.D.

ADDED IN PROOF. It has been pointed out to me that Lemma 2.1 may be found in Schwarz [S, §6]. What is new here is Lemma 2.2, and its application.

\section{BIBLIOGRAPHY}

[N] A. Neeman, GAGA for quotient varieties (to appear).

[GAGA] J. P. Serre, Géométrie algébrique et géométrie analytique, Ann. Inst. Fourier Grenoble 6 (1956), $1-42$.

[S] G. Schwarz, Lifting smooth homotopies of orbit spaces, Publ. Math. Inst. Hautes Etudes Sci. 51 (1980), 37-136.

Department of Mathematics, Princeton University, Princeton, New Jersey 08544 